B. Choe, H. Koo and K. Na

Nagoya Math. J.

Vol. 185 (2007), 31-62

\title{
POSITIVE TOEPLITZ OPERATORS OF SCHATTEN-HERZ TYPE
}

\author{
BOORIM CHOE, HYUNGWOON KOO AND KYUNGUK NA*
}

\begin{abstract}
Motivated by a recent work of Loaiza et al. for the holomorphic case on the disk, we introduce and study the notion of Schatten-Herz type Toeplitz operators acting on the harmonic Bergman space of the ball. We obtain characterizations of positive Toeplitz operators of Schatten-Herz type in terms of averaging functions and Berezin transforms of symbol functions. Our characterization in terms of Berezin transforms settles a question posed by Loaiza et al.
\end{abstract}

\section{$\S 1$. Introduction}

For a fixed integer $n \geq 2$, let $B=B_{n}$ denote the open unit ball in $\mathbf{R}^{n}$. The harmonic Bergman space $b^{2}=b^{2}(B)$ is the set of all complex-valued harmonic functions $f$ on $B$ such that

$$
\|f\|_{2}=\left\{\int_{B}|f|^{2} d V\right\}^{1 / 2}<\infty
$$

where $V$ denotes the Lebesgue volume measure on $B$. For simplicity, we use the notation $d y=d V(y)$, etc.

For $1 \leq p \leq \infty$, let $L^{p}=L^{p}(V)$ be the Lebesgue spaces on $B$. As is well known, $b^{2}$ is a closed subspace of $L^{2}$ and hence is a Hilbert space. By the mean value property of harmonic functions, it is easily seen that point evaluations are continuous on $b^{2}$. Thus, to each $x \in B$, there corresponds a unique $R(x, \cdot) \in b^{2}$ which has the following reproducing property:

$$
f(x)=\int_{B} f(y) \overline{R(x, y)} d y, \quad x \in B
$$

for all $f \in b^{2}$. The explicit formula of the kernel function $R(x, y)$ is well

Received May 6, 2005.

2000 Mathematics Subject Classification: Primary 47B35; Secondary 46E30.

*This research was supported by KOSEF(R01-2003-000-10243-0) 
known:

$$
R(x, y)=\frac{1}{|B|} \cdot \frac{1}{[x, y]^{n}}\left\{\left(\frac{1-|x|^{2}|y|^{2}}{[x, y]}\right)^{2}-\frac{4|x|^{2}|y|^{2}}{n}\right\}
$$

for $x, y \in B$ where $[x, y]=\sqrt{1-2 x \cdot y+|x|^{2}|y|^{2}}$. Here, as in elsewhere, we write $x \cdot y$ for the dot product of $x, y \in \mathbf{R}^{n}$ and $|E|=V(E)$ for the volume of Borel sets $E \subset B$. In particular, the kernel function $R(x, y)$ is real and hence the complex conjugation in the integral of (1.1) can be removed. See [1] for more information and related facts.

Let $Q$ be the Hilbert space orthogonal projection from $L^{2}$ onto $b^{2}$. The reproducing property (1.1) yields the following integral representation of $Q$ :

$$
Q \psi(x)=\int_{B} \psi(y) R(x, y) d y, \quad x \in B
$$

for functions $\psi \in L^{2}$. Since the function $R(x, \cdot)$ and its derivatives, when $x$ stays away from the boundary, are easily seen to be uniformly bounded on $B$, the projection $Q$ can be extended to an integral operator via (1.3) from $L^{1}$ into the space of all harmonic functions on $B$. It even extends to $\mathcal{M}$, the space of all complex Borel measures on $B$. Namely, for each $\mu \in \mathcal{M}$, the integral

$$
Q \mu(x)=\int_{B} R(x, y) d \mu(y), \quad x \in B
$$

defines a function harmonic on $B$.

For $\mu \in \mathcal{M}$, the Toeplitz operator $T_{\mu}$ with symbol $\mu$ is defined by

$$
T_{\mu} f=Q(f d \mu)
$$

for $f \in b^{2} \cap L^{\infty}$. In case $d \mu=\varphi d V$, we write $T_{\mu}=T_{\varphi}$. Note that $T_{\mu}$ is defined on a dense subset of $b^{2}$, because bounded harmonic functions form a dense subset of $b^{2}$; see, for example, Lemma 2.5 of [2].

A Toeplitz operator $T_{\mu}$ is called positive if $\mu \in \mathcal{M}$ is a positive (finite) Borel measure (we will simply write $\mu \geq 0$ ). For positive Toeplitz operators, it is known that many basic properties in the holomorphic case continue to hold for the harmonic case. See [2], [3], [4] and [10]. For example, the characterizations (see Propositions 2.4 and 2.5) of boundedness, compactness and membership of the Schatten classes for a positive Toeplitz operator are complete analogues of characterizations in the holomorphic case obtained by K. Zhu [12]. 
Recently, M. Loaiza, M. López-García and S. Pérez-Esteva [9] studied another aspect of positive Toeplitz operators in the holomorphic case on the unit disk. Roughly speaking, they decomposed a given positive Toeplitz operator into a family of local operators, introduced mixed norm spaces associated with Schatten classes and then gave characterizations of membership in those spaces in terms of the so-called Herz spaces. Motivated by such results in the holomorphic case, we obtain in this paper the harmonic analogues on the ball.

In order to state our results we introduce some notation first. Throughout the paper we let

$$
A_{m}=\left\{x \in B: r_{m} \leq|x|<r_{m+1}\right\}
$$

where $r_{m}=1-2^{-m}$ for each integer $m \geq 0$. We will write $\chi_{m}$ for the characteristic function of $A_{m}$ for each $m$. Also, given $\mu \in \mathcal{M}$, we let $\mu \chi_{m}$ stand for the restriction of $\mu$ to $A_{m}$ for each $m$. Let $1 \leq p, q \leq \infty$ and $\mu \in \mathcal{M}$. Let $S_{p}$ denote the Schatten $p$-class described in Section 2. We say that $T_{\mu}$ is a Schatten-Herz $(p, q)$-type operator if $T_{\mu \chi_{m}} \in S_{p}$ for each $m$ and the sequence $\left\{\left\|T_{\mu \chi_{m}}\right\|_{S_{p}}\right\}$ belongs to $\ell^{q}$. We will write $S_{p, q}$ for the space of all Schatten-Herz $(p, q)$-type operators.

In this paper we give characterizations for a positive Toeplitz operator to be of Schatten-Herz type. In view of Proposition 2.4 below one may naturally expect such characterizations to be in terms of certain mixed norm spaces involving averaging functions or Berezin transforms. Such mixed norm spaces turn out to be the so-called Herz spaces $\mathcal{K}_{q}^{p}(\lambda)$ consisting of all measurable functions $f$ on $B$ such that the sequence $\left\{\left\|f \chi_{m}\right\|_{L^{p}(\lambda)}\right\}$ belongs to $\ell^{q}$. Here, and in what follows, we let $\lambda$ denote the measure on $B$ defined by

$$
d \lambda(x)=R(x, x) d x .
$$

Also, we refer to Section 2 for definitions of the averaging functions $\widehat{\mu}_{\delta}$ and the Berezin transform $\widetilde{\mu}$ of $\mu \in \mathcal{M}$.

The following theorem is our main result.

Theorem 1.1. Let $1 \leq p, q \leq \infty, \delta \in(0,1)$ and $\mu \geq 0$. Then the following conditions are equivalent:

(a) $T_{\mu} \in S_{p, q}$.

(b) $\widetilde{\mu} \in \mathcal{K}_{q}^{p}(\lambda)$. 
(c) $\widehat{\mu}_{\delta} \in \mathcal{K}_{q}^{p}(\lambda)$.

Remark. The holomorphic version of the equivalence (a) $\Longleftrightarrow$ (c) above was proved on the disk in [9]. While our method for the proof of (a) $\Longleftrightarrow(\mathrm{c})$ is basically adapted from [9], substantial and nontrivial amount of extra work is required for the setting of harmonic Bergman spaces. In the same paper [9], however, the holomorphic version of the equivalence (a) $\Longleftrightarrow$ (b) was proved only for the case $p=1$ or $p=q$ and the case $q \neq p>1$ has been left open. One can easily modify our argument of the present paper to remove such a restriction as above.

In Section 2 we review some basic notions such as Berezin transform, averaging functions, Schatten class operators and Herz spaces. In Section 3 we prove various mapping properties of the Berezin transform which will be the main tool in our argument. In Section 4 we prove our main result Theorem 1.1 and observe some consequences.

We often abbreviate inessential constants involved in inequalities by writing $X \lesssim Y$ for positive quantities $X$ and $Y$ if the ratio $X / Y$ has a positive upper bound. Also, we write $X \approx Y$ if $X \lesssim Y$ and $Y \lesssim X$.

\section{$\S 2$. Preliminaries}

In this section we briefly review some basic notions and their properties.

We first recall Berezin transform and averaging functions. Let $\mu \in \mathcal{M}$. Its Berezin transform $\widetilde{\mu}$ is then a function on $B$ defined by

$$
\widetilde{\mu}(x)=\int_{B}|r(x, y)|^{2} d \mu(y), \quad x \in B
$$

where

$$
r(x, \cdot)=\frac{R(x, \cdot)}{\|R(x, \cdot)\|_{2}}
$$

is the normalized reproducing kernel. For $\varphi \in L^{1}$, we define $\widetilde{\varphi}=\widetilde{\mu}$ where $d \mu=\varphi d V$. The notion of Berezin transform can be extended even to nonintegrable functions which belongs to some weighted Lebesgue spaces. See Proposition 3.6 below.

For $\delta \in(0,1)$ fixed, the averaging function $\widehat{\mu}_{\delta}$ is defined by

$$
\widehat{\mu}_{\delta}(x)=\frac{\mu\left[E_{\delta}(x)\right]}{\left|E_{\delta}(x)\right|}, \quad x \in B
$$


where

$$
E_{\delta}(x)=\{y \in B:|x-y|<\delta(1-|x|)\}
$$

is the euclidean ball with center at $x$ and radius $\delta(1-|x|)$. Also, we let $\widehat{\mu}_{\delta}=\widehat{\varphi}_{\delta}$ for $d \mu=\varphi d V$.

The estimate of the kernel function along the diagonal is easily seen. Namely, it is straightforward from (1.1) and (1.2) to see that

$$
R(x, x)=\|R(x, \cdot)\|_{2}^{2} \approx(1-|x|)^{-n}
$$

for $x \in B$. This estimate continues to hold even for points staying sufficiently close to the diagonal in the sense that there exists some $\delta_{0} \in(0,1)$ for which we have

$$
R(x, y) \approx(1-|x|)^{-n}
$$

whenever $x \in B$ and $y \in E_{\delta_{0}}(x)$; see Lemma 2.3 of [2] on general domains. A consequence useful for our purpose is the fact that averaging functions over balls of small radii are dominated by Berezin transforms.

Proposition 2.1. Given $\delta \in\left(0, \delta_{0}\right)$, there exists a constant $C=C(\delta)$ such that $\widehat{\mu}_{\delta} \leq C \widetilde{\mu}$ for $\mu \geq 0$.

Proof. In order to see this note that we have by (2.1) and (2.2)

$$
|r(x, y)|^{2} \approx(1-|x|)^{-n}, \quad y \in E_{\delta}(x)
$$

and the estimate is uniform in $x \in B$. So, for a given $\mu \geq 0$, we have

$$
\begin{aligned}
\widehat{\mu}_{\delta}(x) & \approx(1-|x|)^{-n} \int_{E_{\delta}(x)} 1 d \mu(y) \\
& \approx \int_{E_{\delta}(x)}|r(x, y)|^{2} d \mu(y) \\
& \leq \int_{B}|r(x, y)|^{2} d \mu(y) \\
& =\widetilde{\mu}(x)
\end{aligned}
$$

as desired. The proof is complete.

For positive measures there are more useful properties of the Berezin transform and the averaging operators. Here, we mention a couple of such properties taken from [2] and [3] where the settings are more general. The following is taken from Lemma 3.2 of [2]. 
Proposition 2.2. Given $\delta, \epsilon \in(0,1)$, there exists a constant $C=$ $C(\delta, \epsilon)$ such that $\widehat{\mu}_{\delta} \leq C{\widehat{\left(\widehat{\mu}_{\epsilon}\right)}}_{\delta}$ for $\mu \geq 0$.

The following is implicit in the proof of Proposition 2.14 of [3].

Proposition 2.3. Given $\delta \in(0,1)$, there exists a constant $C=C(\delta)$ such that $\widetilde{\mu} \leq C \widetilde{\widehat{\mu}_{\delta}}$ for $\mu \geq 0$.

We now turn to Schatten class operators. For a compact operator $T$ on $b^{2}$, let $\left\{s_{m}(T)\right\}$ be the nonzero eigenvalues with multiplicity of $|T|=$ $\left(T^{*} T\right)^{1 / 2}$ arranged so that the sequence is non-increasing, where $T^{*}$ denotes the Hilbert space adjoint of $T$. This sequence is called the singular value sequence of $T$. For $1 \leq p<\infty$, we say that $T$ is a Schatten $p$-class operator if the singular value sequence $\left\{s_{m}(T)\right\}$ belongs to $\ell^{p}$. Let $S_{p}$ be the space of all Schatten $p$-class operators on $b^{2}$. The space $S_{p}$ is then a Banach space equipped with the norm

$$
\|T\|_{S_{p}}=\left\{\sum_{m}\left|s_{m}(T)\right|^{p}\right\}^{1 / p} .
$$

See [13], for example, for more information and related facts. Also, we denote by $S_{\infty}$ the class of all bounded linear operators on $b^{2}$ and let $\|T\|_{S_{\infty}}$ denote the operator norm $\|T\|$ of $T \in S_{\infty}$.

The following characterization for a positive Toeplitz operator to be a member of the class $S_{p}$ is taken from Theorems 3.9 and 3.13 of [2] where the setting is more general. Note that the case $p=\infty$ gives characterizations for boundedness, which is also included in Proposition 2.5 below. We did so for easier reference later.

Proposition 2.4. Let $1 \leq p \leq \infty, \delta \in(0,1)$ and $\mu \geq 0$. Then the following conditions are equivalent:

(a) $T_{\mu} \in S_{p}$.

(b) $\tilde{\mu} \in L^{p}(\lambda)$.

(c) $\widehat{\mu}_{\delta} \in L^{p}(\lambda)$.

Moreover, the equivalences $\left\|T_{\mu}\right\|_{S_{p}} \approx\|\widetilde{\mu}\|_{L^{p}(\lambda)} \approx\left\|\widehat{\mu}_{\delta}\right\|_{L^{p}(\lambda)}$ hold. 
We also have corresponding characterizations for boundedness (compactness resp.) taken from Theorems 3.5 and 3.9 (Theorems 3.11 and 3.12 resp.) of [2]. Here, $L_{0}$ denotes the space of all bounded functions $f$ on $B$ such that $f(x) \rightarrow 0$ as $|x| \rightarrow 1$.

Proposition 2.5. Let $\delta \in(0,1)$ and $\mu \geq 0$. Then the following conditions are equivalent:

(a) $T_{\mu}$ is bounded (compact) on $b^{2}$.

(b) $\widetilde{\mu} \in L^{\infty}\left(L_{0}\right)$.

(c) $\widehat{\mu}_{\delta} \in L^{\infty}\left(L_{0}\right)$.

(d) The inclusion $J_{\mu}: b^{2} \subset L^{2}(\mu)$ is bounded (compact).

Moreover, the equivalences $\left\|T_{\mu}\right\| \approx\|\widetilde{\mu}\|_{L^{\infty}} \approx\left\|\widehat{\mu}_{\delta}\right\|_{L^{\infty}} \approx\left\|J_{\mu}\right\|^{2}$ hold.

This proposition has the following consequence which is actually true on the general setting of [2]. Since it is not mentioned in [2] explicitly, we include a proof.

Proposition 2.6. Let $\mu \in \mathcal{M}$ and assume that $T_{|\mu|}$ is bounded on $b^{2}$. Then $T_{\mu}$ is bounded on $b^{2}$ and

$$
\left\|T_{\mu}\right\| \leq C\left\|T_{|\mu|}\right\|
$$

for some constant $C$ independent of $\mu$. If $T_{|\mu|}$ is compact on $b^{2}$ in addition, then $T_{\mu}$ is also compact on $b^{2}$.

Proof. Since $T_{|\mu|}$ is bounded on $b^{2}$ by assumption, the proof of Lemma 3.8 of [2] shows that

$$
\int_{B} \int_{B}|R(x, y)| d x d|\mu|(y)<\infty
$$

and therefore one can apply Fubini's theorem to justify

$$
\int_{B}\left(T_{\mu} f\right) \bar{g} d V=\int_{B} f \bar{g} d \mu
$$


for $f, g \in b^{2} \cap L^{\infty}$. Since the inclusion $J_{|\mu|}: b^{2} \subset L^{2}(|\mu|)$ is bounded by Proposition 2.5, it follows that

$$
\left|\int_{B}\left(T_{\mu} f\right) \bar{g} d V\right| \leq\|f\|_{L^{2}(|\mu|)}\|g\|_{L^{2}(|\mu|)} \leq\left\|J_{|\mu|}\right\|^{2}\|f\|_{2}\|g\|_{2}
$$

for $f, g \in b^{2} \cap L^{\infty}$. Now, since $b^{2} \cap L^{\infty}$ is dense in $b^{2}$, we conclude that $T_{\mu}$ is bounded on $b^{2}$ with $\left\|T_{\mu}\right\| \leq\left\|J_{|\mu|}\right\|^{2}$ and thus the first part of the proposition holds by Proposition 2.5.

Now, suppose that $T_{|\mu|}$ is compact on $b^{2}$. By $(2.3)$ we have

$$
\left\|T_{\mu} f\right\|_{2} \leq\left\|J_{|\mu|}\right\|\|f\|_{L^{2}(|\mu|)}
$$

for $f \in b^{2}$. Thus, given a sequence $\left\{f_{n}\right\}$ in $b^{2}$ which converges weakly to 0 , we obtain

$$
\left\|T_{\mu} f_{n}\right\|_{2} \leq\left\|J_{|\mu|}\right\|\left\|f_{n}\right\|_{L^{2}(|\mu|)} \rightarrow 0, \quad n \rightarrow \infty,
$$

because the inclusion $J_{|\mu|}$ is compact by Proposition 2.5. The proof is complete.

Finally, we recall the Herz spaces on the ball. Let $\alpha$ be real and $1 \leq$ $p, q \leq \infty$. Then the Herz space $\mathcal{K}_{q}^{p, \alpha}$ is the space consisting of all functions $f \in L_{\mathrm{loc}}^{p}(V)$ such that

$$
\|f\|_{\mathcal{K}_{q}^{p, \alpha}}=\left\|\left\{2^{-m \alpha}\left\|f \chi_{m}\right\|_{L^{p}}\right\}\right\|_{\ell^{q}}<\infty ;
$$

recall that $\chi_{m}$ denotes the characteristic function of the annulus $A_{m}$. Equipped with the norm above, the space $\mathcal{K}_{q}^{p, \alpha}$ is a Banach space. Note that an application of Hölder's inequality yields

$$
\int_{B} f \bar{g} d V=\sum_{m} \int_{A_{m}} f g d V \leq \sum_{m}\left\|f \chi_{m}\right\|_{L^{p}}\left\|g \chi_{m}\right\|_{L^{p^{\prime}}}
$$

for positive measurable functions $f$ and $g$. Here, and in what follows, $p^{\prime}$ denotes the conjugate exponent of $p$. Now, another application of Hölder's inequality leads to Hölder's inequality for the Herz spaces as follows:

$$
\left|\int_{B} f \bar{g} d V\right| \leq\|f\|_{\mathcal{K}_{q}^{p, \alpha}}\|g\|_{\mathcal{K}_{q^{\prime}}^{p^{\prime},-\alpha}}, \quad f \in \mathcal{K}_{q}^{p, \alpha}, \quad g \in \mathcal{K}_{q^{\prime}}^{p^{\prime},-\alpha}
$$

for the full range $1 \leq p, q \leq \infty$ and arbitrary $\alpha$ real. We remark in passing that this Hölder's inequality actually leads to dualities between Herz spaces; see Theorem 2.1 and Corollary 2.7 of [7] for details. 
Note that, given $1 \leq p \leq \infty$, we have $\left\|\chi_{m}\right\|_{L^{p}} \approx 2^{-m / p}$ for $m \geq 0$. Thus, the space $\mathcal{K}_{q}^{p, \alpha}$ contains constant functions if and only if either (i) $\alpha>-1 / p$ or (ii) $\alpha \geq-1 / p$ and $q=\infty$. Thus, if either (i) $\alpha<1 / p^{\prime}$ or (ii) $\alpha \leq 1 / p^{\prime}$ and $q=1$, then we have by $(2.4)$

$$
\int_{B}|f| d V \leq\|f\|_{\mathcal{K}_{q}^{p, \alpha}}\|1\|_{\mathcal{K}_{q^{\prime}}^{p^{\prime},-\alpha}}<\infty
$$

for $f \in \mathcal{K}_{q}^{p, \alpha}$. In particular, we have $\mathcal{K}_{q}^{p, \alpha} \subset L^{1}$ whenever $\alpha<1 / p^{\prime}$.

Given $\gamma$ real, let $V_{\gamma}$ denote the weighted measure on $B$ defined by

$$
d V_{\gamma}(x)=(1-|x|)^{\gamma} d x
$$

Let $1 \leq p<\infty$ and $\alpha$ real. Then, given $m \geq 0$, we have $1-|x| \approx 2^{-m}$ for $x \in A_{m}$ and thus obtain

$$
\begin{aligned}
2^{-m \alpha}\left\|f \chi_{m}\right\|_{L^{p}} & =\left\{\int_{A_{m}}\left(2^{-m \alpha}|f(x)|\right)^{p} d x\right\}^{1 / p} \\
& \approx\left\{\int_{A_{m}}(1-|x|)^{\alpha p}|f(x)|^{p} d x\right\}^{1 / p} \\
& \approx\left\|f \chi_{m}\right\|_{L^{p}\left(V_{\alpha p}\right)}
\end{aligned}
$$

and this estimate is uniform in $m$. It follows that

$$
\|f\|_{\mathcal{K}_{q}^{p, \alpha}} \approx\left\|\left\{\left\|f \chi_{m}\right\|_{L^{p}\left(V_{\alpha p}\right)}\right\}\right\|_{\ell^{q}}
$$

for $1 \leq q \leq \infty$. In particular, since $\lambda \approx V_{-n}$, we have

$$
\|f\|_{\mathcal{K}_{q}^{p,-n / p}} \approx\left\|\left\{\left\|f \chi_{m}\right\|_{L^{p}(\lambda)}\right\}\right\|_{\ell^{q}}
$$

this estimate is easily seen to be valid even for $p=\infty$. So, equipped with the norm of $\mathcal{K}_{q}^{p,-n / p}$, the space $\mathcal{K}_{q}^{p}(\lambda)$ is precisely the same as $\mathcal{K}_{q}^{p,-n / p}$ for the full range $1 \leq p, q \leq \infty$. Also, note that

$$
\mathcal{K}_{p}^{p}(\lambda) \approx L^{p}(\lambda)
$$

for $1 \leq p \leq \infty$. That is, these two spaces are the same as sets and have equivalent norms as Banach spaces. 


\section{$\S 3$. Berezin Transform}

In this section we prove various mapping properties of the Berezin transform which we need for the proof of Theorem 1.1 in the next section.

Our first observation is that the Berezin transform continuously takes the Herz spaces $\mathcal{K}_{q}^{p}(\lambda)$ into $L^{\infty}$. We begin with the Herz norm estimates of the kernel function. For that purpose, we recall boundary integral estimates of the kernel function. Note that each function $R(x, \cdot), x \in B$, has a continuous extension up to the boundary and $|R(x, \zeta)| \lesssim[x, \zeta]^{-n}=|x-\zeta|^{-n}$ for $\zeta \in \partial B$. Motivated by this, we put

$$
J_{\beta}(x)=\int_{\partial B} \frac{d S(\zeta)}{|x-\zeta|^{n+\beta}}
$$

for $x \in B$ and $\beta$ real. Here, and in what follows, $S$ denotes the surface area measure on $\partial B$. For $\beta>-1$, it is known that

$$
J_{\beta}(x) \lesssim \frac{1}{(1-|x|)^{\beta+1}}, \quad x \in B
$$

see Lemma 3.2.(d) of [5].

Lemma 3.1. Let $1 \leq p, q \leq \infty$ and assume $-1 / p<\alpha<2 n-n / p$. Then there exists a constant $C=C(\alpha, p, q)$ such that

$$
\left\|R_{x}^{2}\right\|_{\mathcal{K}_{q}^{p, \alpha}} \leq \frac{C}{(1-|x|)^{2 n-n / p-\alpha}}, \quad R_{x}=R(x, \cdot)
$$

for $x \in B$.

Proof. Fix $x \in B$. We first estimate $\left\|R_{x}^{2} \chi_{m}\right\|_{L^{p}}$. Note that $R(x, r \zeta)=$ $R(r x, \zeta)$ for $\zeta \in \partial B$ and $0<r<1$. Thus, for $p<\infty$, we have by (3.2)

$$
\begin{aligned}
\left\|R_{x}^{2} \chi_{m}\right\|_{L^{p}}^{p} & =\int_{A_{m}}|R(x, y)|^{2 p} d y \\
& =\int_{r_{m}}^{r_{m+1}} r^{n-1} \int_{\partial B}|R(r x, \zeta)|^{2 p} d S(\zeta) d r \\
& \lesssim \int_{r_{m}}^{r_{m+1}} J_{2 p n-n}(r x) d r \\
& \lesssim \frac{r_{m+1}-r_{m}}{\left(1-r_{m+1}|x|\right)^{2 p n-n+1}} \\
& \lesssim \frac{2^{-m}}{\left(1-|x|+2^{-m}|x|\right)^{2 p n-n+1}} .
\end{aligned}
$$


We therefore conclude

$$
\left\|R_{x}^{2} \chi_{m}\right\|_{L^{p}} \lesssim \frac{2^{-m / p}}{\left(1-|x|+2^{-m}|x|\right)^{2 n-(n-1) / p}}
$$

and this estimate is uniform in $m$. Also, this estimate remains valid for $p=\infty$, because $|R(x, y)| \lesssim[x, y]^{-n} \leq(1-|x||y|)^{-n}$ by (1.2).

Now, we estimate the Herz norm of $R_{x}^{2}$. Let $1 \leq q \leq \infty$. For $|x| \leq 1 / 2$, it is easily seen from (3.3) that

$$
\left\|R_{x}^{2}\right\|_{\mathcal{K}_{q}^{p, \alpha}} \lesssim\left\|\left\{2^{-m(\alpha+1 / p)}\right\}\right\|_{\ell^{q}} \approx 1 .
$$

So, let $|x|>1 / 2$. First, if $(1-|x|) \leq 2^{-m}$, then we have by (3.3)

$$
2^{-m \alpha}\left\|R_{x}^{2} \chi_{m}\right\|_{L^{p}} \lesssim \frac{2^{-m(\alpha+1 / p)}}{2^{-m(2 n-(n-1) / p)}}=2^{m(2 n-n / p-\alpha)} \leq \frac{1}{(1-|x|)^{2 n-n / p-\alpha}} .
$$

Meanwhile, if $(1-|x|)>2^{-m}$, then we have by (3.3)

$$
2^{-m \alpha}\left\|R_{x}^{2} \chi_{m}\right\|_{L^{p}} \lesssim \frac{2^{-m(\alpha+1 / p)}}{(1-|x|)^{2 n-(n-1) / p}}<\frac{1}{(1-|x|)^{2 n-n / p-\alpha}} .
$$

So, the case $q=\infty$ follows. Also, for $q<\infty$, it follows from these estimates that

$$
\begin{aligned}
\left\|R_{x}^{2}\right\|_{\mathcal{K}_{q}^{p, \alpha}}^{q} & =\sum_{m} 2^{-m q \alpha}\left\|R_{x}^{2} \chi_{m}\right\|_{L^{p}}^{q} \\
& \lesssim \sum_{m \leq \log _{2}(1-|x|)^{-1}} 2^{m q(2 n-n / p-\alpha)} \\
& \quad+\frac{1}{(1-|x|)^{q(2 n-(n-1) / p)}} \sum_{m>\log _{2}(1-|x|)^{-1}} 2^{-m q(\alpha+1 / p)} \\
& \lesssim \frac{1}{(1-|x|)^{q(2 n-n / p-\alpha)}},
\end{aligned}
$$

as desired. The proof is complete.

Recall that if $\alpha<1 / p^{\prime}$, then $\mathcal{K}_{q}^{p, \alpha} \subset L^{1}$. In particular, the Berezin transform is well defined on the space $\mathcal{K}_{q}^{p}(\lambda) \subset L^{1}$ for $1 \leq p, q \leq \infty$.

Proposition 3.2. Let $1 \leq p, q \leq \infty$. There exists a constant $C=$ $C(p, q)$ such that

$$
\|\widetilde{\varphi}\|_{L^{\infty}} \leq C\|\varphi\|_{\mathcal{K}_{q}^{p}(\lambda)}
$$

for functions $\varphi \in \mathcal{K}_{q}^{p}(\lambda)$. 
Proof. Let $\varphi \in \mathcal{K}_{q}^{p}(\lambda)$ and $x \in B$. We may assume $\varphi \geq 0$. Recall that $R(x, x) \approx(1-|x|)^{-n}$. Thus, by (2.4) and Lemma 3.1 with $\alpha=-n / p$, we obtain

$$
\begin{aligned}
\widetilde{\varphi}(x) & \approx(1-|x|)^{n} \int_{B} \varphi(y)|R(x, y)|^{2} d y \\
& \leq(1-|x|)^{n}\|\varphi\|_{\mathcal{K}_{q}^{p,-n / p}}\left\|R_{x}^{2}\right\|_{\mathcal{K}_{q^{\prime}}^{p^{\prime}, n / p}} \\
& \lesssim\|\varphi\|_{\mathcal{K}_{q}^{p,-n / p}} \frac{(1-|x|)^{n}}{(1-|x|)^{2 n-n / p^{\prime}-n / p}} \\
& =\|\varphi\|_{\mathcal{K}_{q}^{p,-n / p}} .
\end{aligned}
$$

Now, since $\mathcal{K}_{q}^{p}(\lambda)=\mathcal{K}_{q}^{p,-n / p}$, we conclude the proposition.

As a corollary we see that Toeplitz operators with $\mathcal{K}_{q}^{p}(\lambda)$-symbols are compact when $q$ is finite.

Corollary 3.3. Let $1 \leq p \leq \infty, 1 \leq q<\infty$ and $\varphi \in \mathcal{K}_{q}^{p}(\lambda)$. Then $T_{\varphi}$ is compact on $b^{2}$.

Proof. Given an integer $N \geq 0$, let $\psi_{N}=\varphi\left(\chi_{0}+\cdots+\chi_{N}\right)$ and $\tau_{N}=$ $\left|\varphi-\psi_{N}\right|$. Then, by Propositions 2.6, 2.5 and 3.2, we have

$$
\left\|T_{\varphi}-T_{\psi_{N}}\right\| \lesssim\left\|T_{\tau_{N}}\right\| \approx\left\|\widetilde{\tau_{N}}\right\|_{L^{\infty}} \lesssim\left\|\tau_{N}\right\|_{\mathcal{K}_{q}^{p}(\lambda)}=\left\|\varphi-\psi_{N}\right\|_{\mathcal{K}_{q}^{p}(\lambda)} .
$$

Meanwhile, since $\left\|\varphi \chi_{m}\right\|_{L^{p}(\lambda)} \approx\left\|\varphi \chi_{m}\right\|_{\mathcal{K}_{q}^{p}(\lambda)}$ for all $m$, we have

$$
\left\|\varphi-\psi_{N}\right\|_{\mathcal{K}_{q}^{p}(\lambda)}^{q} \approx \sum_{m \geq N+1}\left\|\varphi \chi_{m}\right\|_{\mathcal{K}_{q}^{p}(\lambda)}^{q} \rightarrow 0
$$

as $N \rightarrow \infty$ (recall $q<\infty$ ). Accordingly, we see that $T_{\psi_{N}} \rightarrow T_{\varphi}$ in the operator norm. Note that each operator $T_{\psi_{N}}$ is compact on $b^{2}$, because its symbol is supported in a compact subset of $B$. Thus $T_{\varphi}$ is compact on $b^{2}$. The proof is complete.

We now turn to the boundedness of the Berezin transform on the spaces $\mathcal{K}_{q}^{p, \alpha}$ for a certain range of parameters. As a preliminary step we first establish the boundedness of the Berezin transform on the weighted Lebesgue spaces $L^{p}\left(V_{\gamma}\right)$. We actually prove a more general version. We need a couple of lemmas. 
Given $x \in \bar{B}$ with $x \neq 0$, let $\bar{x}=x /|x|^{2}$ stand for the inversion of $x$ with respect to $\partial B$ and define a non-tangential type approach region $\Gamma(x)$ by

$$
\Gamma(x)=\{y \in B:|\bar{x}-y|<\nu(|\bar{x}|-|y|)\}
$$

where $\nu$ is an arbitrary but fixed number such that $1<\nu<3 / 2 \sqrt{2}$. Note that $|\bar{x}|=1 /|x|$.

Lemma 3.4. There is a constant $C=C(n)$ such that

$$
R(x, y) \geq \frac{C}{|\bar{x}-y|^{n}}
$$

for $x, y \in B$ such that $|\bar{x}|-|y| \leq 1 / 5$ and $y \in \Gamma(x)$.

Proof. First, note that

$$
[x, y]=|| \bar{x}|x-| x|y|=|x||\bar{x}-y| \leq|\bar{x}-y|
$$

and therefore we have by $(1.2)$

$$
|B||\bar{x}-y|^{n} R(x, y) \geq\left(\frac{1-|x|^{2}|y|^{2}}{|\bar{x}-y|}\right)^{2}-\frac{4}{n}
$$

for $x, y \in B$.

Now, let $x, y \in B$ be arbitrary points such that $|\bar{x}|-|y| \leq 1 / 5$ and $y \in \Gamma(x)$. Then, since $|\bar{x}|-1<|\bar{x}|-|y| \leq 1 / 5$, we have

$$
\frac{5}{6}(|\bar{x}|-|y|) \leq|x|(|\bar{x}|-|y|)=1-|x||y| \leq \frac{1}{5}
$$

and thus

$$
1-|x|^{2}|y|^{2}=(1-|x||y|)(1+|x||y|) \geq \frac{3}{2}(|\bar{x}|-|y|)>\frac{3}{2 \nu}|\bar{x}-y| .
$$

This, together with (3.4), implies the lemma, because $(3 / 2 \nu)^{2}>4 / n$ by our choice of $\nu$. The proof is complete.

Lemma 3.5. For $-1<\alpha<\infty$ and $c$ real, let

$$
I_{\alpha, c}(x)=\int_{B}|R(x, y)|^{1+(\alpha+c) / n}(1-|y|)^{\alpha} d y
$$


for $x \in B$. Then the following estimates hold:

$$
I_{\alpha, c}(x) \approx \begin{cases}1 & \text { if } c<0 \\ \log \frac{1}{1-|x|} & \text { if } c=0 \\ \frac{1}{(1-|x|)^{c}} & \text { if } c>0\end{cases}
$$

as $|x| \rightarrow 1$.

Proof. Let $-1<\alpha<\infty$ and $c$ real be given. Fix an arbitrary point $x \in B$. We first prove the lower estimates. We only need to consider $x$ sufficiently close to $\partial B$ so that $|\bar{x}|-1<\min \left\{1-\nu^{-1},(10 \nu)^{-1}\right\}$. Put $\eta=x /|x|$. Let

$$
G(x)=\{\zeta \in \partial B: \zeta+\bar{x} \in \Gamma(x)\}
$$

and

$$
G(\eta)=\{\zeta \in \partial B: \zeta+\eta \in \Gamma(\eta)\}
$$

Note that

$$
|\zeta+\bar{x}|<2\left(1-\nu^{-1}\right), \quad \zeta \in G(x)
$$

by definition of the set $G(x)$. Let $G^{*}(x)$ be the convex hull of $G(x)$ and the origin. Also, let

$$
\Omega(x)=\left\{z \in G^{*}(x): 2 \nu(|\bar{x}|-1) \leq|z|<1 / 5\right\} .
$$

We need some properties relevant to these sets. First, we have inclusions

$$
G(\eta) \subset G(x), \quad \bar{x}+\Omega(x) \subset \Gamma(x) \cap B(\bar{x})
$$

where $B(\bar{x})$ is the ball with center at $\bar{x}$ and radius $1 / 5$. To see the first inclusion, let $\zeta \in G(\eta)$. Note that (3.5) remains valid with $\eta$ in place of $x$. So, we have

$$
|\zeta+\bar{x}| \leq|\zeta+\eta|+|\bar{x}-\eta|<2\left(1-\nu^{-1}\right)+|\bar{x}|-1<3\left(1-\nu^{-1}\right)<1
$$

and

$$
|\zeta|<\nu(1-|\zeta+\eta|)=\nu(|\bar{x}|-|\bar{x}-\eta|-|\zeta+\eta|) \leq \nu(|\bar{x}|-|\bar{x}+\zeta|)
$$


so that $\zeta \in G(x)$. To see the second inclusion, given $\zeta \in G(x)$, let $t_{\zeta} \in(0,1)$ be the number such that $t_{\zeta} \zeta+\bar{x} \in \partial B$. Then we have $t \zeta+\bar{x} \in B$ for $t_{\zeta}<t \leq 1$. Note that we have

$$
\begin{aligned}
t|\zeta| & =t|(\zeta+\bar{x})-\bar{x}| \\
& <t \nu(|\bar{x}|-|\zeta+\bar{x}|) \\
& =\nu(|\bar{x}|-t|\zeta+\bar{x}|-(1-t)|\bar{x}|) \\
& \leq \nu(|\bar{x}|-|t \zeta+\bar{x}|)
\end{aligned}
$$

for $0<t \leq 1$. Thus, $t \zeta+\bar{x} \in \Gamma(x)$ for $t_{\zeta}<t \leq 1$. So, since $t_{\zeta}=\left|t_{\zeta} \zeta\right|<$ $\nu(|\bar{x}|-1)$, we have $t G(x)+\bar{x} \subset \Gamma(x)$ for $\nu(|\bar{x}|-1) \leq t<1$, which implies the desired inclusion.

Next, we claim

$$
1-|y| \approx|\bar{x}-y|, \quad y \in \bar{x}+\Omega(x) .
$$

One direction is clear, because $1-|y| \leq|\bar{x}|-|y| \leq|\bar{x}-y|$. To see the other direction, pick any $y \in \bar{x}+\Omega(x) \cap B(\bar{x})$ and put $y-\bar{x}=t \zeta$ where $\zeta \in G(x)$ and $2 \nu(|\bar{x}|-1) \leq t<1 / 5$. Note that $-\eta \in G(x)$ and thus $|\zeta+\eta|<4\left(1-\nu^{-1}\right)$ by (3.5). It follows that

$$
|y| \leq|y-\bar{x}+t \eta|+|\bar{x}|-t=|\bar{x}|-t(1-|\zeta+\eta|)<|\bar{x}|-t\left(4 \nu^{-1}-3\right)
$$

and thus

$$
1-|y| \geq t\left(4 \nu^{-1}-3\right)-(|\bar{x}|-1) \geq(7-6 \nu)(|\bar{x}|-1) .
$$

Now, since $y \in \Gamma(x)$ by (3.6), it follows that

$$
|\bar{x}-y|<\nu(|\bar{x}|-|y|) \lesssim(1-|x|)+(1-|y|) \lesssim 1-|y|
$$

as desired. 
Now, by Lemma 3.4, (3.6) and (3.7), we have

$$
\begin{aligned}
I_{\alpha, c}(x) & \geq \int_{\Gamma(x) \cap B(\bar{x})} \frac{(1-|y|)^{\alpha}}{|\bar{x}-y|^{n+\alpha+c}} d y \\
& \gtrsim \int_{\bar{x}+\Omega(x)} \frac{d y}{|\bar{x}-y|^{n+c}} \\
& =\int_{\Omega(x)} \frac{d z}{|z|^{n+c}} \\
& =S[G(x)] \int_{2 \nu(|\bar{x}|-1)}^{1 / 5} \frac{d r}{r^{1+c}} \\
& \geq S[G(\eta)] \int_{2 \nu(|\bar{x}|-1)}^{1 / 5} \frac{d r}{r^{1+c}}
\end{aligned}
$$

as $|x| \rightarrow 1$; recall that $S$ denotes the surface area measure on $\partial B$. This yields the desired lower estimates.

We now turn to the upper estimates. Note that if $I_{\alpha, c^{\prime}}$ is bounded on $B$ for some $c^{\prime}<0$, then so is $I_{\alpha, c}$ for every $c<c^{\prime}$. So, it suffices to consider $c>-1$; another proof for $c>0$ can be found in Lemma 3.2.(c) of [5]. Let $J_{\beta}$ denote the integral defined in (3.1). Then, integrating in polar coordinates and then manipulating the resulting integral, we have

$$
I_{\alpha, c}(x) \lesssim \int_{0}^{1}(1-t)^{\alpha} J_{\alpha+c}(t x) d t \lesssim 1+\int_{0}^{1}(1-t)^{\alpha+1} J_{\alpha+c+1}(t x) d t ;
$$

the second inequality holds by integration by parts and the estimate $\left|\frac{\partial}{\partial t}\left[J_{\alpha+c}(t x)\right]\right| \leq 2 J_{\alpha+c+1}(t x)$. Now, since $\alpha+1>0$, the integral in the rightmost side of the above is dominated by

$$
\int_{0}^{1} J_{c}(t x) d t \lesssim \int_{0}^{1} \frac{1}{(1-t|x|)^{c+1}} d t
$$

where the inequality holds by (3.2), because $c>-1$. It is now elementary to see that the last integral above satisfies the desired estimates for $c>-1$. The proof is complete.

Given $\alpha$ and $\beta$ real, we let

$$
\Phi_{\alpha, \beta} f(x)=(1-|x|)^{\alpha} \int_{B} f(y)|R(x, y)|^{1+(\alpha+\beta) / n} d V_{\beta}(y), \quad x \in B
$$


whenever the integral is well defined. A complete range of parameters, which ensures the boundedness of these operators on weighted Lebesgue spaces, is given as in the next proposition. Note that $\Phi_{n, 0}$ is equivalent to the Berezin transform.

Proposition 3.6. Let $1 \leq p<\infty$ and $\alpha, \beta$, $\gamma$ be real. Then $\Phi_{\alpha, \beta}$ is bounded on $L^{p}\left(V_{\gamma}\right)$ if and only if $-p \alpha<\gamma+1<p(\beta+1)$.

The holomorphic version of the above proposition is well known. See Theorem 1.9 of [6]. We here included the proof below, which is a modification of the proof of Theorem 1.9 of [6], for completeness.

Proof. We first prove the necessity. Suppose $\Phi_{\alpha, \beta}$ is bounded on $L^{p}\left(V_{\gamma}\right)$. Apply $\Phi$ to a function of the form $f(x)=(1-|x|)^{N}$, where $N$ is chosen sufficiently large so that $N>\alpha$ and $N>-\beta-1$. Then we have

$$
\Phi_{\alpha, \beta} f(x) \approx(1-|x|)^{\alpha}, \quad x \in B
$$

by Lemma 3.5. Since $\Phi_{\alpha, \beta} f \in L^{p}\left(V_{\gamma}\right)$ by assumption, the above yields the inequality $p \alpha+\gamma>-1$. It remains to prove the inequality $\gamma+1<p(\beta+1)$. First, consider the case $p>1$ and let $\Phi_{\alpha, \beta}^{*}$ be the adjoint operator of $\Phi_{\alpha, \beta}$ with respect to the dual action induced by the inner product of $L^{2}\left(V_{\gamma}\right)$. A little manipulation yields the relation $\Phi_{\alpha, \beta}^{*}=\Phi_{\beta-\gamma, \alpha+\gamma}$. Note that $\Phi_{\alpha, \beta}^{*}$ is bounded on $L^{p^{\prime}}\left(V_{\gamma}\right)$. Thus, applying the same reasoning as above, we have the inequality $p^{\prime}(\beta-\gamma)+\gamma>-1$, or equivalently, $p(\beta+1)>\gamma+1$, as desired. Next, consider the case $p=1$. We then need to show the inequality $\beta>\gamma$. Note that $\Phi_{\alpha, \beta}^{*}$ is bounded on $L^{\infty}\left(V_{\gamma}\right)$. In particular, $\Phi_{\alpha, \beta}^{*} 1$ is a bounded function on $B$. More explicitly, we have

$$
\sup _{x \in B}(1-|x|)^{\beta-\gamma} \int_{B}|R(x, y)|^{1+(\alpha+\beta) / n}(1-|y|)^{\alpha+\gamma} d y<\infty .
$$

This, together with Lemma 3.5, yields $\beta>\gamma$ as desired and the proof of the necessity is complete.

We now prove the sufficiency. So, assume $-p \alpha<\gamma+1<p(\beta+1)$. The case $p=1$ is an easy consequence of Lemma 3.5 and Fubini's theorem. So, assume $p>1$. Since

$$
\begin{aligned}
\gamma+1<p(\beta+1) & \Longleftrightarrow-\frac{\beta+1}{p^{\prime}}<\frac{\beta-\gamma}{p} \\
-p \alpha<\gamma+1 & \Longleftrightarrow-\frac{\alpha+\gamma+1}{p}<\frac{\alpha}{p^{\prime}}
\end{aligned}
$$


we see that the intersection of intervals

$$
\left(-\frac{\beta+1}{p^{\prime}}, \frac{\alpha}{p^{\prime}}\right) \bigcap\left(-\frac{\alpha+\gamma+1}{p}, \frac{\beta-\gamma}{p}\right)
$$

is nonempty. Fix any $s$ in this intersection so that

$$
\beta+p^{\prime} s>-1, \quad \alpha-p^{\prime} s>0, \quad \alpha+p s+\gamma>-1, \quad \beta-p s-\gamma>0 .
$$

Thus, by Lemma 3.5, we have

$$
\begin{aligned}
& \int_{B}(1-|y|)^{\beta+p^{\prime} s}|R(x, y)|^{1+(\alpha+\beta) / n} d y \lesssim(1-|x|)^{-\alpha+p^{\prime} s}, \quad x \in B \\
& \int_{B}(1-|x|)^{\alpha+p s+\gamma}|R(x, y)|^{1+(\alpha+\beta) / n} d x \lesssim(1-|y|)^{-\beta+p s+\gamma}, \quad y \in B .
\end{aligned}
$$

Now, setting

$$
K(x, y)=(1-|x|)^{\alpha}(1-|y|)^{\beta-\gamma}|R(x, y)|^{1+(\alpha+\beta) / n}
$$

which is the kernel for the operator $\Phi_{\alpha, \beta}$ against the measure $V_{\gamma}(y)$, we can rewrite the above estimates as

$$
\begin{array}{ll}
\int_{B}(1-|y|)^{p^{\prime} s} K(x, y) d V_{\gamma}(y) \lesssim(1-|x|)^{p^{\prime} s}, & x \in B \\
\int_{B}(1-|x|)^{p s} K(x, y) d V_{\gamma}(x) \lesssim(1-|y|)^{p s}, & y \in B
\end{array}
$$

the suppressed constants in these estimates are independent of $x, y \in B$. We now conclude the boundedness of $\Phi_{\alpha, \beta}$ on $L^{p}\left(V_{\gamma}\right)$ by Schur's test (see, for example, Theorem 1.8 of [13]). The proof is complete.

Remark. In case $(\alpha+\beta) / n$ is an integer, let

$$
\Lambda_{\alpha, \beta} f(x)=(1-|x|)^{\alpha} \int_{B} f(y) R(x, y)^{1+(\alpha+\beta) / n} d V_{\beta}(y), \quad x \in B
$$

whenever the integral is well defined. Then, as in Theorem 1.9 of [13], one can see that, for $1 \leq p<\infty$, the parameter range for the boundedness of $\Lambda_{\alpha, \beta}$ on $L^{p}\left(V_{\gamma}\right)$ is also precisely $-p \alpha<\gamma+1<p(\beta+1)$. In particular, the special case $\alpha=\beta=\gamma=0$ shows that the projection $Q$ is bounded on $L^{p}$ if and only if $1<p<\infty$; also, see [11].

Next, we need an interpolation property of operators on the Herz spaces. Let $\mathfrak{M}_{+}$be the class of all positive (possibly infinite) measurable functions on $B$. 
Lemma 3.7. Let $1 \leq p<\infty, 1 \leq q \leq \infty$ and $\alpha$ be real. Assume that a mapping $T: \mathfrak{M}_{+} \rightarrow \mathfrak{M}_{+}$satisfies the following conditions for functions $f, g$ and sequences $\left\{f_{j}\right\}$ in $\mathfrak{M}_{+}$:

(i) $T(f+g) \leq T f+T g$.

(ii) $T\left(\liminf f_{j}\right) \leq \liminf T f_{j}$.

(iii) $\|T f\|_{L^{p}\left(V_{\alpha p \pm \epsilon}\right)} \leq C_{1}\|f\|_{L^{p}\left(V_{\alpha p \pm \epsilon}\right)}$ for some constants $\epsilon, C_{1}>0$ independent of $f$.

Then there is a constant $C$ such that

$$
\|T f\|_{\mathcal{K}_{q}^{p, \alpha}} \leq C\|f\|_{\mathcal{K}_{q}^{p, \alpha}}
$$

for $f \in \mathfrak{M}_{+}$.

Proof. Let $f \in \mathfrak{M}_{+}$. By (iii) we have

$$
\int_{B}\left|T\left(f \chi_{m}\right)\right|^{p} d V_{\alpha p \pm \epsilon} \leq C_{1} \int_{A_{m}}|f|^{p} d V_{\alpha p \pm \epsilon}
$$

for each $m \geq 0$. Note that $1-|x| \approx 2^{-m}$ for $x \in A_{m}$ and $m \geq 0$. Thus the above estimate yields

$$
\begin{aligned}
\int_{A_{k}}\left|T\left(f \chi_{m}\right)\right|^{p} d V_{\alpha p} & \approx 2^{\mp \epsilon k} \int_{A_{k}}\left|T\left(f \chi_{m}\right)\right|^{p} d V_{\alpha p \pm \epsilon} \\
& \lesssim 2^{\mp \epsilon k} \int_{A_{m}}\left|f \chi_{m}\right|^{p} d V_{\alpha p \pm \epsilon} \\
& \approx 2^{\mp \epsilon(k-m)} \int_{B}\left|f \chi_{m}\right|^{p} d V_{\alpha p}
\end{aligned}
$$

so that

$$
\left\|T\left(f \chi_{m}\right) \chi_{k}\right\|_{L^{p}\left(V_{\alpha p}\right)} \lesssim 2^{-\epsilon|k-m| / p}\left\|f \chi_{m}\right\|_{L^{p}\left(V_{\alpha p}\right)}
$$

for all integers $m, k \geq 0$.

Now, since $T\left(\sum_{m=1}^{N} f \chi_{m}\right) \leq \sum_{m=1}^{N} T\left(f \chi_{m}\right)$ for each $N$ by (i), we have $T f \leq \sum_{m} T\left(f \chi_{m}\right)$ by (ii). So, for each $k=0,1, \cdots$, we have by (3.8)

$$
\left\|(T f) \chi_{k}\right\|_{L^{p}\left(V_{\alpha p}\right)} \leq \sum_{m}\left\|T\left(f \chi_{m}\right) \chi_{k}\right\|_{L^{p}\left(V_{\alpha p}\right)} \lesssim \sum_{m} 2^{-\epsilon|k-m| / p}\left\|f \chi_{m}\right\|_{L^{p}\left(V_{\alpha p}\right)}
$$


and therefore conclude by (2.5)

$$
\|T f\|_{\mathcal{K}_{q}^{p, \alpha}} \lesssim\left\{\sum_{k} 2^{-\epsilon k / p}\right\}\left\|\left\{\left\|f \chi_{m}\right\|_{L^{p}\left(V_{\alpha p}\right)}\right\}\right\|_{\ell^{q}} \approx\|f\|_{\mathcal{K}_{q}^{p, \alpha}}
$$

where the first inequality holds by Young's inequality. The proof is complete.

We are now ready to prove the following $\mathcal{K}_{q}^{p, \alpha}$-boundedness of the Berezin transform.

Proposition 3.8. Let $1 \leq p<\infty, 1 \leq q \leq \infty$ and $\alpha$ be real. If $-n-1 / p<\alpha<1 / p^{\prime}$, then the Berezin transform is bounded on $\mathcal{K}_{q}^{p, \alpha}$. In particular, the Berezin transform is bounded on $\mathcal{K}_{q}^{p}(\lambda)$.

Proof. Assume $-n-1 / p<\alpha<1 / p^{\prime}$, or equivalently, $-n p<\alpha p+1<p$. Note that the Berezin transform is well defined on $\mathcal{K}_{q}^{p, \alpha} \subset L^{1}$, because $\alpha<1 / p^{\prime}$. Also, note that the Berezin transform takes $\mathfrak{M}_{+}$into itself. Thus, for boundedness on $\mathcal{K}_{q}^{p, \alpha}$, it is sufficient to show that the Berezin transform, when restricted to $\mathfrak{M}_{+}$, satisfies all the three conditions in the hypothesis of Lemma 3.7. First, the conditions (i) and (ii) hold by linearity and Fatou's lemma. Next, choosing an $\epsilon>0$ such that

$$
-n p<\alpha p \pm \epsilon+1<p,
$$

we see by Proposition 3.6 (with $\alpha=n$ and $\beta=0$ ) that the Berezin transform is actually bounded on $L^{p}\left(V_{\alpha p \pm \epsilon}\right)$. So, the condition (iii) also holds. This completes the proof of the first part of proposition. Taking $\alpha=-n / p$, we have the second part of the proposition. The proof is complete.

The second part of Proposition 3.8 remains true even for $p=\infty$. See Corollary 4.5.

\section{§4. Positive Toeplitz operators in $S_{p, q}$}

In this section we prove our main result Theorem 1.1 and observe some consequences. Given $1 \leq p, q \leq \infty$, recall that the space $S_{p, q}$ consists of all Toeplitz operators $T_{\mu}$ of Schatten-Herz $(p, q)$-type, meaning that $T_{\mu \chi_{m}} \in S_{p}$ for each $m$ and the sequence $\left\{\left\|T_{\mu \chi_{m}}\right\|_{S_{p}}\right\}$ belongs to $\ell^{q}$. The norm of $T_{\mu} \in$ $S_{p, q}$ is given by

$$
\left\|T_{\mu}\right\|_{S_{p, q}}=\left\|\left\{\left\|T_{\mu \chi_{m}}\right\|_{S_{p}}\right\}\right\|_{\ell^{q}} .
$$

We begin with a simple covering lemma. 
Lemma 4.1. Given $\delta \in(0,1)$, there exists an integer $N=N(\delta)$ such that

$$
E_{\delta}(x) \subset \bigcup_{k=m-1}^{m+N} A_{k} \quad\left(A_{-1}=\emptyset\right)
$$

for $x \in A_{m}$ and $m \geq 0$.

Proof. Fix $\delta \in(0,1)$ and let $N$ be an integer such that $(1-\delta) 2^{N-1} \geq 1$. Let $m \geq 1$ and $x \in A_{m}$. Consider an arbitrary point $y \in E_{\delta}(x)$. Then we have

$$
|y|<|x|+\delta(1-|x|)=1-(1-|x|)(1-\delta)<1-\left(1-r_{m+1}\right) 2^{1-N}=r_{m+N}
$$

and

$$
|y|>|x|-\delta(1-|x|)=1-(1-|x|)(1+\delta) \geq 1-2\left(1-r_{m}\right)=r_{m-1}
$$

for $m \geq 1$. So, we see that $E_{\delta}(x)$ is contained in the annulus with inner radius $r_{m-1}$ and outer radius $r_{m+N}$. For $m=0$ one may easily modify this argument. The proof is complete.

Next, we need a certain sup-norm estimates of the Berezin transforms on each piece of the annuli. For that purpose we introduce more notation. In what follows we let

$$
A_{m, \ell}^{\delta}(\zeta)=A_{m} \bigcap\left[B\left(\zeta, \ell \delta 2^{-m}\right) \backslash B\left(\zeta,(\ell-1) \delta 2^{-m}\right)\right]
$$

for $m \geq 0, \ell \geq 1, \delta \in(0,1)$ and $\zeta \in \partial B$. Here, $B(\zeta, t)=\emptyset$ for $t \leq 0$ and $B(\zeta, t), t>0$, denotes the euclidean ball with center at $\zeta$ and radius $t$. Also, we let $\Sigma(\zeta, t)=B(\zeta, t) \cap \partial B$. Note that $\eta \in \Sigma(\zeta, t)$ if and only if $1-t^{2} / 2<\zeta \cdot \eta \leq 1$. Thus, by slice integration (see, for example, Corollary A.5 of [1]), we obtain

$$
S[\Sigma(\zeta, t)]=c_{n} \int_{1-\frac{t^{2}}{2}}^{1}\left(1-r^{2}\right)^{\frac{n-3}{2}} d r, \quad 0<t \leq 2
$$

where $c_{n}$ is the surface area of $(n-2)$-dimensional unit sphere; recall that $S$ denotes the surface area measure on $\partial B$. 
Given $A_{m, \ell}^{\delta}(\zeta)$, pick a maximal collection of points $x_{m, \ell, 1}, \ldots, x_{m, \ell, M_{m \ell}}$ in $A_{m, \ell}^{\delta}(\zeta)$ subject to the separation condition $E_{\delta / 4}\left(x_{m, \ell, i}\right) \cap E_{\delta / 4}\left(x_{m, \ell, j}\right)=\emptyset$ for $i \neq j$. Then we have

$$
A_{m, \ell}^{\delta}(\zeta) \subset \bigcup_{j=1}^{M_{m \ell}} E_{\delta}\left(x_{m, \ell, j}\right)
$$

by maximality. In order to see this, let $x \in A_{m, \ell}^{\delta}(\zeta)$. Maximality then provides a common point $y$ of some $E_{\delta / 4}\left(x_{m, \ell, j}\right)$ and $E_{\delta / 4}(x)$. Since $1-|x| \leq$ $2^{-m}<2\left(1-\left|x_{m, \ell, j}\right|\right)$, we have with such $y$

$$
\begin{aligned}
\left|x-x_{m, \ell, j}\right| & \leq|x-y|+\left|y-x_{m, \ell, j}\right| \\
& <\frac{\delta}{4}\left(1-|x|+1-\left|x_{m, \ell, j}\right|\right) \\
& <\delta\left(1-\left|x_{m, \ell, j}\right|\right)
\end{aligned}
$$

so that $x \in E_{\delta}\left(x_{m, \ell, j}\right)$. So, (4.2) holds.

By rotation invariance it is clear that $M_{m \ell}$, the number of points introduced above, is independent of $\zeta$. What we need is a uniform estimate for upper bounds of $M_{m \ell}=M_{m \ell}(\delta)$ as in the following.

Lemma 4.2. There exists a positive integer $N_{1}=N_{1}(n)$ such that

$$
M_{m \ell}(\delta) \leq N_{1} \delta^{-n} \ell^{n-2}
$$

whenever $m \geq 0, \ell \geq 1, \delta \in(0,1)$ and $\zeta \in \partial B$.

Proof. Let $m \geq 0, \ell \geq 1, \delta \in(0,1)$ and $\zeta \in \partial B$ be given. We continue using notation introduced in (4.2). Note that

$$
\left.E_{\delta}\left(x_{m, \ell, j}\right) \subset B\left(\zeta,(\ell+1) \delta 2^{-m}\right) \backslash B\left(\zeta,(\ell-2) \delta 2^{-m}\right)\right)
$$

for each $j$ and thus

$$
\bigcup_{j=1}^{M_{m \ell}} E_{\delta}\left(x_{m, \ell, j}\right) \subset \bigcup_{k=m-1}^{m+N} \bigcup_{j=\ell-1}^{\ell+1} A_{k, j}^{\delta}(\zeta)
$$

where $N=N(\delta)$ is the integer provided by Lemma 4.1. We will derive the desired estimate by a standard volume argument using the inclusion above. So, we need to estimate the volume of the set $A_{m, \ell}^{\delta}(\zeta)$. Put

$$
\Sigma_{m, \ell}^{\delta}(\zeta)=\Sigma\left(\zeta,\left(\ell+\delta^{-1}\right) \delta 2^{-m}\right) \backslash \Sigma\left(\zeta,\left(\ell-1-\delta^{-1}\right) \delta 2^{-m}\right)
$$


and let $D_{m, \ell}^{\delta}(\zeta)$ be the set of all $y=|y| \eta \in A_{m}$ where $\eta \in \Sigma_{m, \ell}^{\delta}(\zeta)$. Then it is easily seen that $A_{m, \ell}^{\delta}(\zeta) \subset D_{m, \ell}^{\delta}(\zeta)$.

We first estimate the surface area of the set $\Sigma_{m, \ell}^{\delta}(\zeta)$. Note that $\Sigma_{m, \ell}^{\delta}(\zeta)=$ $\emptyset$ for $2<\left(\ell-1-\delta^{-1}\right) \delta 2^{-m}$. For $0<\left(\ell-1-\delta^{-1}\right) \delta 2^{-m} \leq 2$, we have by $(4.1)$

$$
\begin{aligned}
S\left[\Sigma_{m, \ell}^{\delta}(\zeta)\right] & \lesssim\left(\delta 2^{-m}\right)^{(n-1)}\left[\left(\ell+\delta^{-1}\right)^{n-1}-\left(\ell-1-\delta^{-1}\right)^{n-1}\right] \\
& \approx 2^{-m(n-1)} \ell^{n-2} .
\end{aligned}
$$

Also, for $\left(\ell-1-\delta^{-1}\right) \delta 2^{-m} \leq 0$, we have

$$
S\left[\Sigma_{m, \ell}^{\delta}(\zeta)\right] \lesssim\left(\delta 2^{-m}\right)^{(n-1)}\left(\ell+\delta^{-1}\right)^{n-1} \lesssim 2^{-m(n-1)} \ell^{n-2}
$$

because $\ell \leq 1+\delta^{-1}$. In summary, we have

$$
S\left[\Sigma_{m, \ell}^{\delta}(\zeta)\right] \lesssim 2^{-m(n-1)} \ell^{n-2}
$$

and the constant suppressed in the above inequality depends only on $n$. So, we have

$$
\left|A_{m, \ell}^{\delta}(\zeta)\right| \leq\left|D_{m, \ell}(\zeta)\right| \approx 2^{-m} \cdot S\left[\Sigma_{m, \ell}^{\delta}(\zeta)\right] \lesssim 2^{-m n} \ell^{n-2}
$$

by integration in polar coordinates. We now deduce from (4.3) and (4.4) that

$$
M_{m \ell} \delta^{n} 2^{-m n} \approx \sum_{j=1}^{M_{m \ell}}\left|E_{\delta / 4}\left(x_{m, \ell, j}\right)\right|=\left|\bigcup_{j=1}^{M_{m \ell}} E_{\delta / 4}\left(x_{m, \ell, j}\right)\right| \lesssim 2^{-m n} \ell^{n-2}
$$

and this estimate is uniform in $m, \ell, \delta$ and $\zeta$. This yields an integer $N_{1}=$ $N_{1}(n)$ with the desired property. The proof is complete.

Now, we prove the sup-norm estimates of the Berezin transforms on each piece of the annuli as in the next lemma.

Lemma 4.3. Given $\delta \in(0,1)$, there exists a constant $C=C(\delta)$ such that

$$
\left\|\widetilde{\mu} \chi_{k}\right\|_{L^{\infty}} \leq C \sum_{m=0}^{\infty} \frac{\left\|\left(\widehat{\mu \chi_{m}}\right)_{\delta}\right\|_{L^{\infty}}}{2^{|m-k|}}, \quad k=0,1, \ldots
$$

for all $\mu \geq 0$. 
Proof. Let $\mu \geq 0$ and $k \geq 0$ be given. Let $x \in A_{k}$ be an arbitrary point. We have

$$
\begin{aligned}
\widetilde{\mu}(x) & =\int_{B}|r(x, y)|^{2} d \mu(y) \\
& \lesssim \int_{B} \frac{(1-|x|)^{n}}{[(1-|x|)+(1-|y|)+|x-y|]^{2 n}} d \mu(y)
\end{aligned}
$$

where the second inequality holds by (2.1) and the estimate

$$
|R(x, y)| \lesssim[(1-|x|)+(1-|y|)+|x-y|]^{-n}, \quad y \in B
$$

see Theorem 1.1 of [8].

Let $x=|x| \zeta$ where $\zeta \in \partial B$. We continue using notation introduced in Lemma 4.2. We will estimate the last integral in (4.5) by using the decomposition

$$
B=\bigcup_{m=0}^{\infty} A_{m}=\bigcup_{m=0}^{\infty} \bigcup_{\ell=1}^{\infty} A_{m, \ell}^{\delta}(\zeta)
$$

With this in mind, note that, given $A_{m, \ell}^{\delta}(\zeta)$, we have

$$
|x-y| \geq|\zeta-y|-|x-\zeta| \geq(\ell-1) \delta 2^{-m}-2^{-k-1}
$$

for $y \in A_{m, \ell}^{\delta}(\zeta)$ and thus

$$
(1-|x|)+(1-|y|)+|x-y| \gtrsim 2^{-k}+\ell 2^{-m}, \quad y \in A_{m, \ell}^{\delta}(\zeta)
$$

for all $m$ and $\ell$, because $1-|x| \approx 2^{-k}$ and $1-|y| \approx 2^{-m}$. Also, note that we have by (4.2) and Lemma 4.2

$$
\begin{aligned}
\mu\left[A_{m, \ell}^{\delta}(\zeta)\right] & \leq \sum_{j=1}^{M_{m \ell}} \mu\left[A_{m} \cap E_{\delta}\left(x_{m, \ell, j}\right)\right] \\
& \lesssim\left\|\left(\widehat{\mu \chi_{m}}\right)_{\delta}\right\|_{L^{\infty}} \sum_{j=1}^{M_{m \ell}}\left|E_{\delta}\left(x_{m, \ell, j}\right)\right| \\
& \approx M_{m \ell} 2^{-m n}\left\|\left(\widehat{\mu \chi_{m}}\right)_{\delta}\right\|_{L^{\infty}} \\
& \lesssim N_{1} \delta^{-n} 2^{-m n} \ell^{n-2}\left\|\left(\widehat{\mu \chi_{m}}\right)_{\delta}\right\|_{L^{\infty}}
\end{aligned}
$$

for all $m$ and $\ell$. 
Now, it follows from (4.5), (4.6), (4.7) and (4.8) that

$$
\begin{aligned}
\widetilde{\mu}(x) & \leq \sum_{m=0}^{\infty} \sum_{\ell=1}^{\infty} \int_{A_{m, \ell}^{\delta}(\zeta)} \frac{(1-|x|)^{n}}{[(1-|x|)+(1-|y|)+|x-y|]^{2 n}} d \mu(y) \\
& \lesssim \sum_{m=0}^{\infty} \sum_{\ell=1}^{\infty} \frac{2^{n(k-m)} \ell^{n-2}}{\left(1+\ell 2^{k-m}\right)^{2 n}}\left\|\left(\widehat{\mu \chi_{m}}\right)_{\delta}\right\|_{L^{\infty}} \\
& =\sum_{m=0}^{\infty} 2^{n(k-m)} J_{m k}\left\|\left(\widehat{\mu \chi_{m}}\right)_{\delta}\right\|_{L^{\infty}}
\end{aligned}
$$

where

$$
J_{m k}=\sum_{\ell=1}^{\infty} \frac{\ell^{n-2}}{\left(1+\ell 2^{k-m}\right)^{2 n}} .
$$

To estimate these sums, we only consider $m \geq k$; the case $m<k$ is simpler. Since $m \geq k$, we have

$$
\begin{aligned}
J_{m k} & \approx \sum_{\ell=1}^{2^{m-k}} \ell^{n-2}+\sum_{\ell=2^{m-k}}^{\infty} \frac{\ell^{n-2}}{\left(\ell 2^{k-m}\right)^{2 n}} \\
& \approx 2^{(n-1)(m-k)}+2^{2 n(m-k)} \int_{2^{m-k}}^{\infty} \frac{d t}{t^{n+2}} \\
& \approx 2^{(n-1)(m-k)} \\
& =\frac{1}{2^{n(k-m)}} \frac{1}{2^{|k-m|}} .
\end{aligned}
$$

This, together with (4.9), yields the desired estimate. The proof is complete.

We now turn to the proof of Theorem 1.1. Here, we actually prove a more precise version including the equivalences of associated norms.

Theorem 4.4. Let $1 \leq p, q \leq \infty, \delta \in(0,1)$ and $\mu \geq 0$. Then the following conditions are equivalent:

(a) $T_{\mu} \in S_{p, q}$.

(b) $\widetilde{\mu} \in \mathcal{K}_{q}^{p}(\lambda)$.

(c) $\widehat{\mu}_{\delta} \in \mathcal{K}_{q}^{p}(\lambda)$.

Moreover, the equivalences $\left\|T_{\mu}\right\|_{S_{p, q}} \approx\|\widetilde{\mu}\|_{\mathcal{K}_{q}^{p}(\lambda)} \approx\left\|\widehat{\mu}_{\delta}\right\|_{\mathcal{K}_{q}^{p}(\lambda)}$ hold. 
Proof. We first show the equivalence (a) $\Longleftrightarrow$ (c). It is sufficient to show the estimate

$$
\left\|\widehat{\mu}_{\delta}\right\|_{\mathcal{K}_{q}^{p}(\lambda)} \approx\left\|T_{\mu}\right\|_{S_{p, q}}
$$

We prove this for $q<\infty$; the case $q=\infty$ is simpler. We use the convention that $A_{m}=\emptyset$ and $\chi_{m}=0$ for a negative integer $m$. Fix $\mu \geq 0$ and choose $N=N(\delta)>3$ as in Lemma 4.1. We then have by Lemma 4.1

$$
\widehat{\mu}_{\delta} \chi_{m} \leq \sum_{k=m-1}^{m+N}\left(\widehat{\mu \chi_{k}}\right)_{\delta}
$$

and thus

$$
\left\|\widehat{\mu}_{\delta} \chi_{m}\right\|_{L^{p}(\lambda)}^{q} \leq(N+2)^{q-1} \sum_{k=m-1}^{m+N}\left\|\left(\widehat{\mu \chi_{k}}\right)_{\delta}\right\|_{L^{p}(\lambda)}^{q}
$$

for all $m \geq 0$. Summing up these estimates, we have

$$
\sum_{m=0}^{\infty}\left\|\widehat{\mu}_{\delta} \chi_{m}\right\|_{L^{p}(\lambda)}^{q} \leq(N+2)^{q} \sum_{k=0}^{\infty}\left\|\left(\widehat{\mu \chi_{k}}\right)_{\delta}\right\|_{L^{p}(\lambda)}^{q} .
$$

Accordingly, we conclude

$$
\left\|\widehat{\mu}_{\delta}\right\|_{\mathcal{K}_{q}^{p}(\lambda)}^{q} \lesssim \sum_{k=0}^{\infty}\left\|\left(\widehat{\mu \chi_{k}}\right)_{\delta}\right\|_{L^{p}(\lambda)}^{q} \approx \sum_{k=0}^{\infty}\left\|T_{\mu \chi_{k}}\right\|_{S_{p}}^{q}=\left\|T_{\mu}\right\|_{S_{p, q}}^{q}
$$

where the second equivalence comes from Proposition 2.4.

We now prove the other direction of the estimate (4.11). Note that $E_{\delta}(x)$ can intersect $A_{m}$ with $m \geq 0$ only when $x \in \cup_{k=m-N}^{m+1} A_{k}$ by Lemma 4.1. Thus, we have

$$
\left(\widehat{\mu \chi_{m}}\right)_{\delta}=\sum_{k=m-N}^{m+1}\left(\widehat{\mu \chi_{m}}\right)_{\delta} \chi_{k} \leq \sum_{k=m-N}^{m+1} \widehat{\mu}_{\delta} \chi_{k}
$$

and thus

$$
\left\|(\widehat{\mu \chi m})_{\delta}\right\|_{L^{p}(\lambda)}^{q} \leq(N+2)^{q-1} \sum_{k=m-N}^{m+1}\left\|\widehat{\mu}_{\delta} \chi_{k}\right\|_{L^{p}(\lambda)}^{q}
$$


for each $m \geq 0$. Summing up these estimates, we have

$$
\sum_{m=0}^{\infty}\left\|\left(\widehat{\mu \chi_{m}}\right)_{\delta}\right\|_{L^{p}(\lambda)}^{q} \leq(N+2)^{q} \sum_{k=0}^{\infty}\left\|\widehat{\mu}_{\delta} \chi_{k}\right\|_{L^{p}(\lambda)}^{q} .
$$

Consequently, reversing the estimate in (4.11), we obtain

$$
\left\|T_{\mu}\right\|_{S_{p, q}}^{q} \lesssim\left\|\widehat{\mu}_{\delta}\right\|_{\mathcal{K}_{q}^{p}(\lambda)}^{q}
$$

and thus we conclude (4.10).

Next, we prove the equivalence (b) $\Longleftrightarrow(\mathrm{c})$. Choose $\epsilon \in\left(0, \delta_{0}\right)$ where $\delta_{0}$ is the number provided by Proposition 2.1. Then, by (4.10) and Proposition 2.1 , we have

$$
\left\|\widehat{\mu}_{\delta}\right\|_{\mathcal{K}_{q}^{p}(\lambda)} \approx\left\|\widehat{\mu}_{\epsilon}\right\|_{\mathcal{K}_{q}^{p}(\lambda)} \lesssim\|\widetilde{\mu}\|_{\mathcal{K}_{q}^{p}(\lambda)}
$$

for the full range $1 \leq p, q \leq \infty$.

On the other hand, by Propositions 2.3 and 3.8, we have

$$
\|\widetilde{\mu}\|_{\mathcal{K}_{q}^{p}(\lambda)} \lesssim\left\|\widetilde{\widetilde{\mu}_{\delta}}\right\|_{\mathcal{K}_{q}^{p}(\lambda)} \lesssim\left\|\widehat{\mu}_{\delta}\right\|_{\mathcal{K}_{q}^{p}(\lambda)}
$$

for $1 \leq p<\infty$ and $1 \leq q \leq \infty$. Also, for $p=q=\infty$, we have $\left\|\widehat{\mu}_{\delta}\right\|_{L^{\infty}} \approx$ $\|\widetilde{\mu}\|_{L^{\infty}}$ by Proposition 2.4 and (2.6). Now, consider the case where $p=\infty$ and $q<\infty$. In this case, we have

$$
\|\widetilde{\mu}\|_{\mathcal{K}_{q}^{\infty}(\lambda)} \approx\left\|\left\{\left\|\widetilde{\mu} \chi_{k}\right\|_{L^{\infty}}\right\}\right\|_{\ell^{q}} \lesssim\left\{\sum_{k=0}^{\infty} 2^{-k}\right\}\left\|\left\{\left\|\left(\widehat{\mu \chi_{m}}\right)_{\delta}\right\|_{L^{\infty}}\right\}\right\|_{\ell^{q}}
$$

by Lemma 4.3 and Young's inequality. Meanwhile, we have

$$
\left\|\left\{\left\|\left(\widehat{\mu \chi_{m}}\right)_{\delta}\right\|_{L^{\infty}}\right\}\right\|_{\ell^{q}} \lesssim\left\{\sum_{k=0}^{\infty}\left\|\widehat{\mu}_{\delta} \chi_{k}\right\|_{L^{\infty}}^{q}\right\}^{1 / q} \approx\left\|\widehat{\mu}_{\delta}\right\|_{\mathcal{K}_{q}^{\infty}(\lambda)}
$$

as in the proof of (a) $\Longleftrightarrow$ (c) above. Combining these estimates, we have $\|\widetilde{\mu}\|_{\mathcal{K}_{q}^{\infty}(\lambda)} \lesssim\left\|\widehat{\mu}_{\delta}\right\|_{\mathcal{K}_{q}^{\infty}(\lambda)}$, as desired. This completes the proof.

We now observe some consequences. Given $\delta \in(0,1)$, it is not hard to see that the averaging operator $\varphi \mapsto \widehat{\varphi}_{\delta}$ is $L^{p}$-bounded for $p=1$ or $p=\infty$ and thus for all $1 \leq p \leq \infty$ by the Riesz-Thorin interpolation theorem. It turns out that the averaging operator is bounded on each of the Herz spaces $\mathcal{K}_{q}^{p}(\lambda)$. Combining this fact with Theorem 4.4, we have the boundedness of the Berezin transform on $\mathcal{K}_{q}^{\infty}(\lambda)$ which is the missing case in Proposition 3.8 . 
Corollary 4.5. Let $1 \leq p, q \leq \infty$ and $\delta \in(0,1)$. Then the averaging operator $\varphi \mapsto \widehat{\varphi}_{\delta}$ is bounded on $\mathcal{K}_{q}^{p}(\lambda)$. Also, the Berezin transform is bounded on $\mathcal{K}_{q}^{p}(\lambda)$.

Proof. Let $\varphi \in \mathcal{K}_{q}^{p}(\lambda)$ and assume $\varphi \geq 0$ without loss of generality. We first consider the case $p=\infty$ and $q<\infty$. We have

$$
\|\widetilde{\varphi}\|_{\mathcal{K}_{q}^{\infty}(\lambda)}^{q} \approx\left\|\widehat{\varphi}_{\delta}\right\|_{\mathcal{K}_{q}^{\infty}(\lambda)}^{q} \lesssim \sum_{k=0}^{\infty}\left\|\left(\widehat{\varphi \chi_{k}}\right)_{\delta}\right\|_{L^{\infty}}^{q} \leq \sum_{k=0}^{\infty}\left\|\varphi \chi_{k}\right\|_{L^{\infty}}^{q} \approx\|\varphi\|_{\mathcal{K}_{q}^{\infty}(\lambda)}^{q}
$$

by Theorem 4.4 and the first inequality in (4.11). All the remaining cases follows from Theorem 4.4 and Proposition 3.8. The proof is complete.

Next, the following is an immediate consequence of (2.6), Proposition 2.4 and Theorem 4.4.

Corollary 4.6. Let $1 \leq p \leq \infty$ and $\mu \geq 0$. Then $T_{\mu} \in S_{p, p}$ if and only if $T_{\mu} \in S_{p}$.

Also, we observe that the operator norm of positive Toeplitz operators are dominated by their $S_{p, q}$-norms. This consequence in turn implies that operators in $S_{p, q}$ are all compact for finite $q$.

Corollary 4.7. Let $1 \leq p, q \leq \infty$. Assume $\mu \in \mathcal{M}$ and $T_{|\mu|} \in S_{p, q}$. Then

$$
\left\|T_{\mu}\right\| \leq C\left\|T_{|\mu|}\right\|_{S_{p, q}}
$$

for some constant $C=C(p, q)$ independent of $\mu$. If $q<\infty$ and $d \mu=\varphi d V$ in addition, then $T_{\mu}$ is compact on $b^{2}$.

Proof. By Proposition 2.6 we may assume $\mu \geq 0$ without loss of generality. First, we have

$$
\left\|T_{\mu}\right\| \approx\left\|\widehat{\mu}_{\delta}\right\|_{L^{\infty}} \lesssim\left\|\widehat{\left(\widehat{\mu}_{\delta}\right)_{\delta}}\right\|_{L^{\infty}}
$$

by Propositions 2.4 and 2.2. Meantime, we have

$$
\left\|\widehat{\left(\widehat{\mu}_{\delta}\right)_{\delta}}\right\|_{L^{\infty}} \approx\left\|\widetilde{\left(\widehat{\mu}_{\delta}\right)}\right\|_{L^{\infty}} \lesssim\left\|\widehat{\mu}_{\delta}\right\|_{\mathcal{K}_{q}^{p}(\lambda)} \approx\left\|T_{\mu}\right\|_{S_{p, q}}
$$

by Propositions 2.4, 3.2 and Theorem 4.4. This proves the first part of the corollary. 
Now suppose that $\varphi \in L^{1}$ and $T_{|\varphi|} \in S_{p, q}$. Given an integer $N \geq 0$, let $\psi_{N}=|\varphi|\left(\chi_{0}+\cdots+\chi_{N}\right)$. Then we have

$$
\left\|T_{|\varphi|}-T_{\psi_{N}}\right\|_{S_{p, q}}^{q}=\sum_{m \geq N+1}\left\|T_{|\varphi| \chi_{m}}\right\|_{S_{p}}^{q}
$$

by definition of $S_{p, q}$-norm. Note that the right side of the above tends to 0 as $N \rightarrow \infty$ for $q<\infty$. So, the second part of the lemma follows as in the proof of Corollary 3.3. The proof is complete.

Finally, we prove that the spaces $S_{p, q}$ are all different by constructing explicit examples.

Corollary 4.8. Let $1 \leq p, q, p_{1}, q_{1} \leq \infty$. Then $S_{p, q}=S_{p_{1}, q_{1}}$ if and only if $p=p_{1}$ and $q=q_{1}$.

Proof. Given $1 \leq a, b \leq \infty$, put

$$
\varphi=\varphi_{a, b}=\sum_{k=1}^{\infty} \frac{\log k}{2^{k(n-1) / a} k^{1 / b}} \chi_{k} .
$$

Let $\delta \in(0,1)$ be a sufficiently small number. For example, one may take $\delta<1 / 5$. Let $1 \leq s \leq \infty$. We first prove

$$
\left\|\widehat{\varphi}_{\delta} \chi_{m}\right\|_{L^{s}(\lambda)} \approx \frac{\log m}{2^{m(n-1)(1 / a-1 / s)} m^{1 / b}}
$$

for integers $m \geq 2$.

Let $m \geq 2$ and put

$$
A_{m}^{*}=\left\{y \in B: r_{m}+\frac{1}{3} \cdot \frac{1}{2^{m+1}} \leq|y|<r_{m+1}-\frac{1}{3} \cdot \frac{1}{2^{m+1}}\right\} .
$$

Note that $\lambda\left(A_{m}^{*}\right) \approx 2^{m n}\left|A_{m}^{*}\right| \approx 2^{m(n-1)}$. Similarly, $\lambda\left(A_{m}\right) \approx 2^{m(n-1)}$. Also, since $\delta<1 / 5$, it is easily checked that $E_{\delta}(x) \subset A_{m}$ for $x \in A_{m}^{*}$ as in the proof of Lemma 4.1. It follows that $\left(\widehat{\chi_{m}}\right)_{\delta}=1$ on $A_{m}^{*}$ and thus

$$
\left\|\left(\widehat{\chi_{m}}\right)_{\delta} \chi_{m}\right\|_{L^{s}(\lambda)} \geq \lambda\left(A_{m}^{*}\right)^{1 / s} \approx 2^{m(n-1) / s}
$$

This yields the lower estimate

$$
\begin{aligned}
\left\|\widehat{\varphi}_{\delta} \chi_{m}\right\|_{L^{s}(\lambda)} & \geq \frac{\log m}{2^{m(n-1) / a} m^{1 / b}}\left\|\left(\widehat{\chi_{m}}\right)_{\delta} \chi_{m}\right\|_{L^{s}(\lambda)} \\
& \gtrsim \frac{\log m}{2^{m(n-1)(1 / a-1 / s)} m^{1 / b}} .
\end{aligned}
$$


We now prove the upper estimate. Since $\left(\widehat{\chi_{k}}\right)_{\delta} \leq 1$ on $B$, we obtain

$$
\left\|\left(\widehat{\chi_{k}}\right)_{\delta} \chi_{m}\right\|_{L^{s}(\lambda)} \leq\left\|\chi_{m}\right\|_{L^{s}(\lambda)}=\lambda\left(A_{m}\right)^{1 / s} \approx 2^{m(n-1) / s}
$$

for $k \geq 1$. Also, we have $E_{\delta}(x) \subset \bigcup_{k=m-1}^{m+2} A_{k}$ for $x \in A_{m}$ by Lemma 4.1. Accordingly, we conclude

$$
\begin{aligned}
\left\|\widehat{\varphi}_{\delta} \chi_{m}\right\|_{L^{s}(\lambda)} & \leq \sum_{k=m-1}^{m+2} \frac{\log k}{2^{k(n-1) / a} k^{1 / b}}\left\|\left(\widehat{\chi_{k}}\right)_{\delta} \chi_{m}\right\|_{L^{s}(\lambda)} \\
& \lesssim \frac{\log m}{2^{m(n-1)(1 / a-1 / s)} m^{1 / b}}
\end{aligned}
$$

as desired.

Now, assume that either $p \neq p_{1}$ or $q \neq q_{1}$. We only need to consider two cases:

(i) $p<p_{1}$;

(ii) $p=p_{1}$ and $q<q_{1}$.

By Theorem 4.4 it suffices to prove that

$$
\widehat{\varphi}_{\delta} \in \mathcal{K}_{q_{1}}^{p_{1}}(\lambda), \quad \widehat{\varphi}_{\delta} \notin \mathcal{K}_{q}^{p}(\lambda)
$$

for some choice of $a$ and $b$. Before proceeding, note that $\left\|\widehat{\varphi}_{\delta} \chi_{m}\right\|_{L^{s}(\lambda)}<\infty$ for $m=0,1$. First, consider Case (i). In this case we take $a=p$ and $b=\infty$. Then, for $1 \leq s, t \leq \infty$, it follows from (4.13) that

$$
\widehat{\varphi}_{\delta} \in \mathcal{K}_{t}^{s}(\lambda) \Longleftrightarrow\left\{\frac{\log m}{2^{m(n-1)(1 / p-1 / s)}}\right\} \in \ell^{t} \Longleftrightarrow p<s
$$

and therefore (4.14) holds. Next, consider Case (ii). In this case we take $a=p$ and $b=q$. Then, for $1 \leq t \leq \infty$, we have by (4.13)

$$
\widehat{\varphi}_{\delta} \in \mathcal{K}_{t}^{p}(\lambda) \Longleftrightarrow\left\{\frac{\log m}{m^{1 / q}}\right\} \in \ell^{t} \Longleftrightarrow q<t
$$

so that (4.14) holds. The proof is complete. 


\section{REFERENCES}

[1] S. Axler, P. Bourdon and W. Ramey, Harmonic function theory, 2nd ed., SpringerVerlag, New York, 2001.

[2] B. Choe, Y. Lee and K. Na, Toeplitz operators on harmonic Bergman spaces, Nagoya Math. J., 174 (2004), 165-186.

[3] B. Choe, Y. Lee and K. Na, Positive Toeplitz operators from a harmonic Bergman space into another, Tohoku Math. J., 56 (2004), 255-270.

[4] B. Choe, H. Koo and H. Yi, Positive Toeplitz operators between the harmonic Bergman spaces, Potential Analysis, 17 (2002), 307-335.

[5] R. R. Coifman and R. Rochberg, Representation theorems for holomorphic and harmonic functions, Astérisque, 77 (1980), 11-65.

[6] H. Handenmalm, B. Koremblum and K. Zhu, Theory of Bergman spaces, Springer Verlag, New York, 2000.

[7] E. Herenández and D. Yang, Interpolation of Herz spaces and applications, Math. Nachr., 205 (1999), 69-87.

[8] H. Kang and H, Koo, Estimates of the harmonic Bergman kernel on smooth domains, J. Funct. Anal., 185 (2001), 220-239.

[9] M. Loaiza, M. López-García and S. Pérez-Esteva, Herz classes and Toeplitz operators in the disk, Integral Equations Operator Theory, 53 (2005), 287-296.

[10] J. Miao, Reproducing kenkels for harmonic Bergman spaces of the unit ball, Monatsh. Math., 125 (1998), 25-35.

[11] K. Stroethoff, Harmonic Bergman spaces, Holomorphic spaces, MRSI Publications, 33 (1998), 51-63.

[12] K. Zhu, Positive Toeplitz operators on weighted Bergman spaces of bounded symmetric domains, J. Operator Theory, 20 (1988), 329-357.

[13] K. Zhu, Operator theory in function spaces, Marcel Dekker, New York and Basel, 1989.

Boorim Choe

Department of Mathematics

Korea University

Seoul 136-713, KOREA

cbr@korea.ac.kr

Hyungwoon Koo

Department of Mathematics

Korea University

Seoul 136-713, KOREA

koohw@korea.ac.kr 
Kyunguk Na

Department of Mathematics

Korea University

Seoul 136-713, KOREA

nakyunguk@korea.ac.kr 